\title{
Association and cis-mQTL analysis of variants in serotonergic genes associated with nicotine dependence in Chinese Han smokers
}

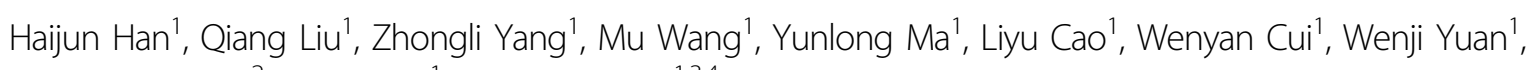
Thomas J. Payne ${ }^{2}$, Lanjuan $\mathrm{Li}^{1}$ and Ming D. Li $\mathrm{i}^{1,3,4}$

\begin{abstract}
Variants in serotonergic genes are implicated in nicotine dependence (ND) in subjects of European and African origin, but their involvement with smoking in Asians is largely unknown. Moreover, mechanisms underlying the ND riskassociated single-nucleotide polymorphisms (SNPs) in these genes are rarely investigated. The Fagerström Test for Nicotine Dependence (FTND) score was used to assess ND in 2616 male Chinese Han smokers. Both association and interaction analysis were used to examine the association of variants in the serotonergic genes with FTND. Further, expression and methylation quantitative trait loci (cis-mQTL) analysis was employed to determine the association of individual SNPs with the extent of methylation of each CpG locus. Individual SNP-based association analysis revealed that rs 1176744 in HTR3B was marginally associated with FTND $(p=0.042)$. Haplotype-based association analysis found that one major haplotype, T-T-A-G, formed by SNPs rs3758987-rs4938056-rs1176744-rs2276305, located in the $5^{\prime}$ region of HTR3B, showed a significant association with FTND ( $p=0.00025)$. Further, a significant genetic interactive effect affecting ND was detected among SNPs rs10160548 in HTR3A, and rs3758987, rs2276305, and rs1672717 in HTR3B ( $p=0.0074)$. Finally, we found four CpG sites (CpG_4543549, CpG_4543464, CpG_4543682, and CpG_4546888) to be significantly associated with three cis-mQTL SNPs (i.e., rs3758987, rs4938056, and rs1176744) located in our detected haplotype within HTR3B. In sum, we showed SNP rs1176744 (Tyr129Ser) to be associated with ND. Together with the SNPs rs3758987 and rs4938056 in HTR3B, they formed a major haplotype, which had significant association with ND. We further showed these SNPs contribute to ND through four methylated sites in HTR3B. All these findings suggest that variants in the serotonergic system play an important role in ND in the Chinese Han population. More importantly, these findings demonstrated that the involvement of this system in ND is through gene-by-gene interaction and methylation.
\end{abstract}

Correspondence: Ming D. Li (ml2km@zju.edu.cn) or (ming.li@shu.edu) or Lanjuan Li (lji@@zju.edu.cn)

${ }^{1}$ State Key Laboratory for Diagnosis and Treatment of Infectious Diseases, The First Affiliated Hospital, Collaborative Innovation Center for Diagnosis and Treatment of Infectious Diseases, Zhejiang University School of Medicine, Hangzhou, China

${ }^{2}$ ACT Center for Tobacco Treatment, Education and Research, Department of Otolaryngology and Communicative Sciences, University of Mississippi Medical Center, Jackson, MS, USA

Full list of author information is available at the end of the article.

These authors contributed equally: Haijun Han, Qiang Liu

\section{Introduction}

Cigarette smoking is still a serious issue all over the world. Each year, smoking causes about six million deaths worldwide, of which more than five million result directly from cigarette smoking ${ }^{1}$. Although smoking prevalence has decreased greatly since $1990^{2}$, the annual number of smoking-related deaths in the world is projected to rise to more than eight million by $2030^{3,4}$. In China, cigarette

\section{(c) The Author(s) 2018}

(c) (i) Open Access This article is licensed under a Creative Commons Attribution 4.0 International License, which permits use, sharing, adaptation, distribution and reproduction cc) in any medium or format, as long as you give appropriate credit to the original author(s) and the source, provide a link to the Creative Commons license, and indicate if changes were made. The images or other third party material in this article are included in the article's Creative Commons license, unless indicated otherwise in a credit line to the material. If material is not included in the article's Creative Commons license and your intended use is not permitted by statutory regulation or exceeds the permitted use, you will need to obtain permission directly from the copyright holder. To view a copy of this license, visit http://creativecommons.org/licenses/by/4.0/. 
consumption was approximately twofold higher in 2016 than it was in $1998^{5}$, and the prevalence of smoking differs greatly in men and women in different regions ${ }^{6,7}$. A recent report showed that there is a high smoking prevalence in Chinese men, and most of these users are highly nicotinedependent $(\mathrm{ND})^{8}$.

Cigarette smoking is a complex behavior that is influenced by both environmental and genetic factors ${ }^{9}$. Nicotine is one of the primary ingredients of cigarette smoke and is addictive. Meta-analysis of twin and family studies has found the average heritability of ND to be 0.59 in males and 0.46 in females with an average heritability of 0.56 in both males and females ${ }^{10}$. Nicotine exerts its biological effects by binding to nicotinic acetylcholine receptors (nAChRs) located in various brain regions ${ }^{11}$, where it either stimulates or inactivates them through desensitization ${ }^{12}$. The activation of nAChRs in the striatum stimulates dopamine release after nicotine exposure $^{13,14}$. Nicotine can also induce the release of various other neurotransmitters in the brain, such as catecholamines, serotonin (5-hydroxytryptamine, 5-HT), and peptides $^{15}$.

5-HT is a polyfunctional signaling molecule that acts mainly as a neurotransmitter within both the central and peripheral nervous systems ${ }^{16,17}$. The diverse effects of 5HT are mediated by $5-\mathrm{HT}$ receptors, which are grouped into seven classes (5-HT1 through $5-\mathrm{HT} 7)^{18}$. Most of these are G protein-coupled receptors, but the 5-HT3 receptors belong to the Cys-loop superfamily of pentameric neurotransmitter-gated ion channels ${ }^{19,20}$. The 5HT3 receptors share similarities with acetylcholine, subtype A of $\gamma$-aminobutyric acid and glycine receptors, with about $30 \%$ structural and functional homology ${ }^{21}$. Both 5HT3 receptors and nAChRs exist widely in the central nervous system but are localized to presynaptic and postsynaptic sites ${ }^{22,23}$. Also, nAChRs are co-localized with 5-HT3 serotonin receptors on the same presynaptic striatal nerve terminals regulating dopamine neurotransmission, indicating a convergence of cholinergic and serotonergic systems in the striatum ${ }^{24}$.

To date, five 5-HT3 receptor subunit genes (HTR3A, HTR3B, HTR3C, HTR3D, and HTR3E) have been identified in humans that encode five receptor subunits, 5$\mathrm{HT}_{\mathrm{A}}, 5-\mathrm{HT} 3_{\mathrm{B}}, 5-\mathrm{HT} 3_{\mathrm{C}}, 5-\mathrm{HT} 3_{\mathrm{D}}$, and $5-\mathrm{HT}_{\mathrm{E}}{ }^{19}$. Of these, the $5-\mathrm{HT} 3_{\mathrm{A}}$ and $5-\mathrm{HT} 3_{\mathrm{B}}$ subunits are more commonly investigated. Both human HTR3A and HTR $3 B$ are located within a 90-kb region on chromosome 11q23.1 ${ }^{25}$. SLC6A4 or 5-HTT is an important serotonin transporter gene, which is located on chromosome 17q11.2 and encodes a membrane protein that regulates synaptic 5-HT concentrations through recycling $5-\mathrm{HT}$ from the synaptic cleft $^{26}$.

Serotonergic genes have been extensively investigated in various conditions, such as psychiatric ${ }^{27,28}$ and eating ${ }^{29}$ disorders. As a result of 5-HT3 receptor activation, the serotonin-gated ion channel undergoes rapid depolarization and desensitization, then releases stored neurotransmitter, suggesting an important role of the serotonergic system in neuronal circuitry involved in drug abuse $^{30}$. The serotonergic system was reported to be related to the effects of nicotine, leading to the development of $\mathrm{ND}^{31}$. Recently, there were some genetic studies on serotonergic genes in drug abuse, for instance, smoking $^{32}$, alcohol dependence ${ }^{33-35}$, and other drugs dependencies ${ }^{33,36}$. By using various genetic approaches such as genome-wide linkage, candidate gene association, and genome-wide association analysis, this research has provided insights into the genetic architecture of $\mathrm{ND}^{37}$. However, there have been limited genetic studies on the relation of serotonergic genes to smoking in Chinese populations.

Thus, in this study, we applied various genetic analyses to investigate the role of serotonergic genes in ND in a less commonly investigated population, Chinese Han smokers. We first performed association analysis at both the individual single-nucleotide polymorphism (SNP) and haplotype levels. We then determined interactive effects among serotonergic genes to understand their roles in ND. Finally, to explore the underlying mechanisms of ND, we established a link for those risk variants for ND with differential DNA methylation loci by performing methylation quantitative trait locus (cis-mQTL) analysis.

\section{Materials and methods \\ Samples}

The donors of the samples used in the present study were enrolled from local hospitals in Jincheng and Taiyuan in Shanxi Provinces, China, during 2012-2013 . Because only about $2 \%$ of Chinese women smoke ${ }^{38}$, only male smokers $(N=2616)$ were included in the study. For each subject, detailed information on age, education, income, medical history, environment, and smokingrelated behaviors were collected by trained interviewers. For this study, we used the Fagerström Test for Nicotine Dependence (FTND) score (0-10 scale), one of the most commonly used instruments to assess smoking quantity $(\mathrm{SQ})^{39,40}$, to assess the ND of each smoker. On the basis of previous reports, smokers with an FTND score $\geq 6$ were considered to be highly ND, and those with an FTND of $<6$ were classified as moderately or slightly dependent ${ }^{40,41}$. Supplementary Table 1 shows the distribution of FTND scores of smokers in our sample, which included 1243 heavy and 1373 light smokers. All subjects provided written informed consent for participating in the study, which was approved by the Institutional Review Board of the First Affiliated Hospital of Zhejiang University School of Medicine. 
We used Structure 2.3.4 ${ }^{42}$ to assess population stratification according to the genotyping data of a group of 30 ancestry-informative marker panel. The parameters of simulation were set as 100000 burns-in and 100000 iterations and the $K$ to 2 because the samples were collected from two areas. We pooled those two groups for further analysis, as no population admixture was revealed (Supplementary Figure 1).

\section{SNP selection}

According to the minor allele frequency (MAF) $>0.05$ based on the National Center for Biotechnology Information (NCBI) dbSNP database (http://www.ncbi.nlm. nih.gov/SNP/) and published papers ${ }^{32,36,43}, 8$ SNPs in HTR3A, 8 SNPs in HTR3B, and 1 SNP in SLC6A4 were selected for genotyping. Detailed information about each SNP is shown in Supplementary Table 2. To ensure reliable association results, we selected SNPs according to the following criteria: genotype calling rate $>95 \%$, MAF $>$ 0.01 , and Hardy-Weinberg equilibrium (HWE) $p>0.05$. Under these criteria, SNPs rs1150226 and rs33940208 in HTR3A, and rs11606194, rs17614942, and rs3782025 in $H T R 3 B$ were excluded from the analysis because of their low genotyping quality. After removing these SNPs, 12 SNPs were available for subsequent association analysis.

\section{DNA sample processing and genotyping}

For each participant, peripheral blood was collected, and the genomic DNA was extracted using the Gentra Puregene blood kit according to the manufacturer's protocol. The DNA concentration and optical density were measured using NanoDrop 2000, and a $A_{260} / A_{280}$ ratio of 1.7-1.9 was required prior to genotyping. The starting normalized DNA concentration of each sample was set at $50 \mathrm{ng} / \mu \mathrm{l}$. The QuantStudio ${ }^{\mathrm{ma}}$ OpenArray ${ }^{\circ}$ AccuFill $^{\mathrm{mm}}$ System (Applied Biosystems, Inc.) was used to transfer samples and load the OpenArray ${ }^{\circ}$ plate. After sealing of the plate, the OpenArray case was loaded with OpenArray ${ }^{\oplus}$ Immersion Fluid. Finally, the OpenArray ${ }^{\oplus}$ Plug was inserted into the port, and the plug was twisted clockwise until hand-tight, when it was ready to be loaded into the QuantStudio $^{\text {тu }}$ 12K Flex System (Applied Biosystems). The DNA genotyping was performed using the QuantStudio ${ }^{\mathrm{mm}}$ 12K Flex OpenArray platform, which is based on the TaqMan genotyping assay where two probes were labeled with FAM or VIC fluorescent dye. After 50 cycles of amplification, the fluorescent signals were recorded. Further analysis was performed with Life Technologies TaqMan Genotyper (v. 1.3) software. All SNPs were called using an "autocall" feature; further, a manual inspection of the autocalled data was performed by two independent researchers. As noted, samples with a call rate of $<95 \%$ were removed from subsequent association analysis. The threshold for the genotyping rate of all SNPs was set as $\geq 95 \%$, and the $p$ value for the HWE was set as no $\geq 5 \%$. All procedures and conditions used in this study were essentially the same as what we reported previously on the same samples ${ }^{44,45}$.

\section{DNA methylation dataset}

The DNA methylation dataset used here consisted of samples from 36 smokers and 36 nonsmokers selected from the sample set described above on the basis of the age, sex, and smoking status, which is an ongoing wholegenome bisulfite sequencing project in this laboratory ${ }^{45}$. Briefly, the Illumina HiSeq X Ten platform was applied to conduct DNA methylation sequencing with an average of about 700 million $( \pm 75$ million; SD) 150 -bp paired-end reads per sample. Clean reads were mapped to the hg19 reference genome using Bismark ${ }^{46}$. Then, we combined two strands of information of $\mathrm{CpG}$ sites and excluded CpGs with reads $<5$ or those overlapping with common variants in the Chinese Han genome (MAF > 0.05).

\section{Statistical analysis \\ Individual-based association analysis}

For all 12 individual SNPs in HTR3A, HTR3B, and $S L C 6 A 4$, association analysis with the FTND score was performed using a logistic regression model with the PLINK program (v. 1.07) ${ }^{47}$. The additive genetic model was employed for all analyses with age, working time, and area as covariates. Because the samples were collected from coal miners, and because of the different jobs held by the various subjects, some of them needed to work underground for more than $8 \mathrm{~h} /$ day, whereas others, such as technical persons, worked primarily aboveground. It is thus necessary to consider "working time" as a covariate in the analysis. In addition, the samples were collected from two areas of Shanxi province (i.e., Taiyuan and Jincheng), so area was treated as another covariate. A $p$ value of $<0.05$ was considered statistically significant after correction for multiple testing using the Bonferroni procedure.

\section{Haplotype-based association analysis}

Only major haplotypes with a frequency of $>5 \%$ were included in the analysis. Haploview software (v. 4.2) was used to determine pair-wise linkage disequilibrium and haplotype blocks ${ }^{48,49}$. Association analysis with the FTND score was performed using Haplo Stats (v. 1.7.7) ${ }^{50}$. For the approach used for performing haplotype-based association analysis, please refer to our previous reports ${ }^{43,44}$.

\section{Gene-gene interaction analysis}

Genetic interactive effects of HTR $3 A, H T R 3 B$, and $S L C 6 A 4$ were analyzed using the GPU-based Generalized Multifactor Dimensionality Reduction (GMDR-GPU) program developed by our research team ${ }^{51}$. An exhaustive 
search was performed for all possible 2- to 5-locus combination models. The interactive effects among the 12 SNPs were calculated with FTND score according to a generalized linear model by including age, working time, and area as covariates. Three criteria were used to determine the best gene-gene interaction model: (1) the cross-validation consistency (CVC) statistics for the selected variant combinations; (2) the prediction accuracy; and (3) significant $p$ value, which was determined by $10^{7}$ permutation tests for the selected SNP combinations. The $\mathrm{CVC}$ is the number of times the particular combination is selected from all the training sets. The higher the CVC, the more robust the SNP combination as a predictive interaction model. After identifying the candidate interaction models, their prediction accuracies are calculated by averaging their corresponding testing accuracies among all the data partitions that are not contained in the training sets. The significance or $p$ value is calculated by a permutation test based on the prediction accuracy. Based on $10^{7}$ permutation tests, CVC $>7$ of 10 , prediction accuracy $>55 \%$, and empirical $p$ value $<0.005$ are considered a significant SNP combination.

\section{cis-mQTL analysis}

We conducted cis-mQTL analysis with a similar procedure, as described before ${ }^{45}$. The region for the cismQTL analysis was set within $50 \mathrm{~kb}$ upstream and downstream of each SNP of interest ${ }^{52,53}$. Prior to cismQTL analysis, we removed the low-quality DNA methylation sites, those with a calling rate $<80 \%$. Four SNPs (rs3758987, rs4938056, rs1176744, and rs2276305) formed into a haplotype in HTR3B were chosen for the cis-mQTL association analysis. The Matrix eQTL (v. 2.1.1) R package ${ }^{54}$ was used to test association of the four risk SNPs with the extent of methylation of each CpG site using a linear regression model.

Because there were both smokers and nonsmokers in the cohort yielding the methylation data, we adjusted smoking status and age as covariates to detect the effects of each risk variant with adjacent extents of methylation. Significant associations were defined after Bonferroni correction $\left(p<0.05 / 4788=1.04 \times 10^{-5}\right)$, where 4788 is the total number of association tests for the four SNPs (i.e., 1175 CpG sites for rs1176744, 1173 CpG sites for rs2276305, $1221 \mathrm{CpG}$ sites for rs3758987, and $1219 \mathrm{CpG}$ sites for rs4938056). In addition, we performed a cismQTL analysis within smokers using the same method Table 1.

\section{Results}

Individual SNP-based association analysis

The results from the individual SNP-based association analyses with FTND score are shown in Table 2. Of the 12 SNPs, rs1176744 in HTR3B is the only one showing a
Table 1 Characteristics of samples used in the study

\begin{tabular}{llll}
\hline Characteristic & Number (total/mean) & SD & Range \\
\hline No. of subjects & 2,616 & NA & NA \\
Age (years) & 40.4 & 9.7 & $19-74$ \\
FTND score & 5.45 & 1.41 & $0-10$ \\
\hline
\end{tabular}

FTND Fagerström Test for Nicotine Dependence, SD standard deviation, NA not available

Table 2 Individual SNP-based association analysis with FTND in Chinese Han smokers

\begin{tabular}{|c|c|c|c|c|}
\hline \multirow[t]{2}{*}{ Gene } & \multirow[t]{2}{*}{ SNP ID } & \multirow[t]{2}{*}{ MAF } & \multicolumn{2}{|c|}{ Statistics $^{\mathrm{a}}$} \\
\hline & & & Beta & $p$ Value \\
\hline \multirow[t]{6}{*}{ HTR3A } & rs2276302 & $G(0.10)$ & 0.07 & 0.221 \\
\hline & rs1150220 & A (0.09) & 0.09 & 0.166 \\
\hline & rs1062613 & $\mathrm{T}(0.09)$ & 0.08 & 0.236 \\
\hline & rs1176713 & G (0.24) & 0.05 & 0.283 \\
\hline & rs1985242 & A (0.28) & 0.03 & 0.413 \\
\hline & rs10160548 & G (0.34) & 0.00 & 0.989 \\
\hline \multirow[t]{5}{*}{ HTR3B } & rs1176744 & $C(0.17)$ & -0.10 & 0.042 \\
\hline & rs3758987 & $C(0.17)$ & -0.08 & 0.098 \\
\hline & rs1672717 & G (0.38) & -0.05 & 0.202 \\
\hline & rs2276305 & A $(0.27)$ & -0.04 & 0.294 \\
\hline & rs4938056 & $C(0.41)$ & -0.02 & 0.664 \\
\hline SLC6A4 & rs1042173 & $\mathrm{A}(0.20)$ & 0.05 & 0.330 \\
\hline
\end{tabular}

Age, working time, and area were used as covariates. $p$ Values $<0.05$ are shown in boldface type. The corrected $p$ value after Bonferroni correction for 12 tested SNPs is $0.0042(0.05 / 12)$

MAF minor allele frequency

${ }^{a}$ Additive model was used in the analyses

significant association with FTND score under the additive model $(\beta=-0.10 ; p=0.042)$. However, it was no longer significant after Bonferroni correction for multiple testing. We found no significance of the SNPs in HTR3A and SLC6A4 $(p>0.05)$.

\section{Haplotype-based association analysis}

On the basis of the definition of haplotype block by Gabriel et al. ${ }^{49}$, two blocks within HTR3A and one block within $H T R 3 B$ were revealed (Fig. 1). Haplotype-based association analyses for all major haplotypes (frequency $\geq$ $5 \%$ ) were performed in the three blocks mentioned above with FTND score. There was only one major haplotype, T-T-A-G, formed by SNPs rs3758987, rs4938056, rs1176744, and rs2276305, located in the $5^{\prime}$ region of $H T R 3 B$, with a frequency of $14.4 \%$, which was significantly associated with FTND score under the additive model 
(Hap-score $=3.67 ; p=0.00025)$ (Table 3). This association remained significant after Bonferroni correction for multiple testing. On the other hand, the other two major haplotypes in HTR3A did not show any significant association with the FTND score $(p>0.05)$.

\section{Gene-gene interaction analysis}

As we know, $H T R 3 A, H T R 3 B$, and $S L C 6 A 4$ play critical roles in regulating serotonin signaling. Here we

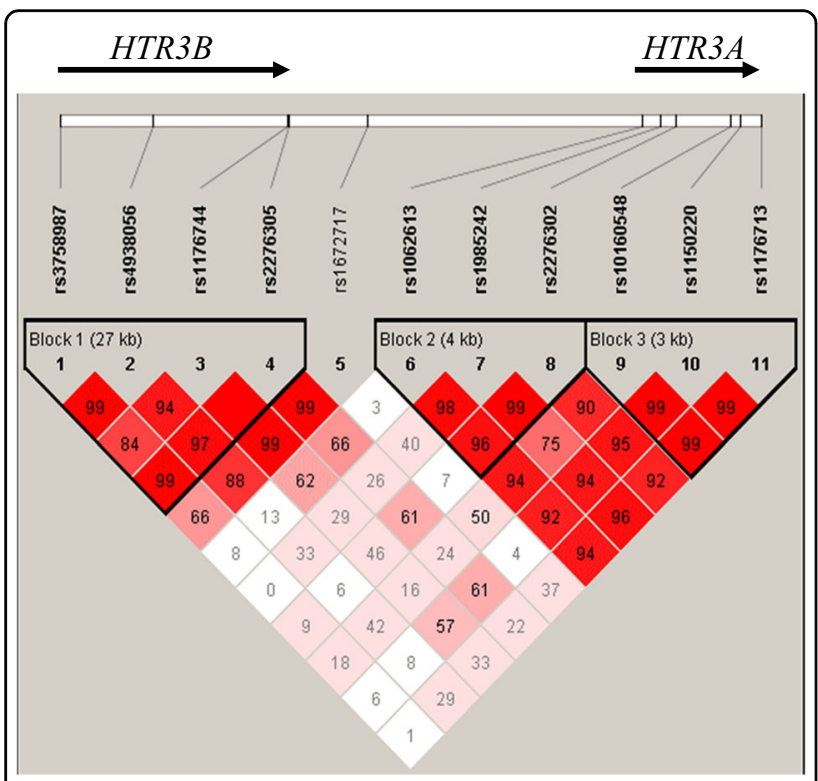

Fig. 1 LD structure for HTR3A and HTR3B SNPs in Chinese Han smokers. The $D^{\prime}$ values were calculated by Haploview (v. 4.2) shown in each box, and haplotype blocks were defined according to the criteria of Gabriel et al. (2002) ${ }^{49}$. The arrow at the top of the figure indicates the gene transcription direction from $5^{\prime}$ to $3^{\prime}$ performed interaction analysis for 12 SNPs on the FTND score. As shown in Table 4, the best model displayed a significant genetic interaction effect with the FTND score. This significant interaction model included rs10160548 in HTR3A, and rs3758987, rs2276305, and rs1672717 in $H T R 3 B$, with a CVC of $8 / 10$, prediction accuracy of $56.41 \%$, and an empirical $p$ value of 0.0074 based on $10^{7}$ permutation tests.

\section{Associations among genotype, DNA methylation, and gene expression}

The associations of the four SNPs in HTR3B with cismQTLs are shown in Supplementary Table 3. Figure 2 depicts the position of the SNPs and CpG sites. Of them, six pairs of significant SNP-CpG associations were detected, which were formed by four SNPs (rs2276305, rs3758987, rs4938056, and rs1176744) and five CpG sites (CpG_4543464, CpG_4541957, CpG_4543549, CpG_4543682, and CpG_4546888), with $p$ values ranging from $1.43 \times 10^{-27}$ to $5.69 \times 10^{-6}$ (Fig. 3 and Supplementary Figure 2).

To confirm the results of cis-mQTL, a similar method was applied to perform cis-mQTL analysis by adjusting with the FTND score and age. The results were in accordance with the above found from the samples of both smokers and nonsmokers (Supplementary Table 2), which further demonstrated that these SNPs are risk variants affecting methylation in smokers.

Because the significant pairs of SNPs and CpG sites are located in HTR3B according to the cis-eQTL association dataset from GTEx Portal (https://gtexportal.org/home/), 12 brain regions were available to test eQTL. We found three (rs3758987, rs1176744, and rs4938056) of the four SNPs showed significant correlation with allele-specific

Table 3 Major haplotypes (frequency $>0.05$ ) within HTR3B and HTR3A associated with FTND in Chinese Han smokers

\begin{tabular}{|c|c|c|c|c|c|c|}
\hline Gene & SNP combination & Haplotype & Hap-Freq & Hap-Score & $p$-Hap & p-Global \\
\hline \multirow[t]{4}{*}{ HTR3B } & rs3758987-rs4938056-rs1176744-rs2276305 & T-T-A-G & 0.14 & 3.67 & 0.00025 & 0.00547 \\
\hline & & C-T-C-G & 0.14 & -1.571 & 0.11535 & \\
\hline & & T-T-A-A & 0.26 & -0.94 & 0.34542 & \\
\hline & & T-C-A-G & 0.40 & -0.38 & 0.70192 & \\
\hline \multirow[t]{7}{*}{ HTR3A } & rs1062613-rs1985242-rs2276302 & T-A-G & 0.09 & 1.28 & 0.20068 & 0.52552 \\
\hline & & C-A-A & 0.18 & -0.08 & 0.93544 & \\
\hline & & C-T-A & 0.71 & -0.94 & 0.34864 & \\
\hline & rs10160548-rs1150220-rs1176713 & G-A-G & 0.10 & 1.35 & 0.17649 & 0.46057 \\
\hline & & G-G-A & 0.10 & -1.28 & 0.1997 & \\
\hline & & G-G-G & 0.14 & -0.16 & 0.87608 & \\
\hline & & T-G-A & 0.66 & 0.04 & 0.97098 & \\
\hline
\end{tabular}

The results were adjusted for age, working time, and area under additive model. Significant association is shown in boldface type. The corrected $p$ value after Bonferroni correction for four tested major haplotypes in HTR3B is $0.0125(0.05 / 4)$ 
Table 4 Best SNP combination in HTR3A and HTR3B associated with FTND in Chinese Han smokers

\begin{tabular}{lllll}
\hline $\begin{array}{l}\text { SNP } \\
\text { combination }\end{array}$ & ND measure & $\begin{array}{l}\text { CVC } \\
\text { Prediction } \\
\text { accuracy }\end{array}$ & $\begin{array}{l}\text { Permutated } \boldsymbol{p} \\
\text { value }\end{array}$ \\
\hline HTR3A: & FTND & $8 / 10$ & $56.41 \%$ & 0.0074 \\
rs10160548 & & & & \\
HTR3B: & & & \\
rs3758987, & & & \\
rs2276305, & & & \\
rs1672717 & & & \\
\hline
\end{tabular}

The empirical $p$ value was calculated by $10^{7}$ permutations CVC cross-validation consistency
mRNA expression of $H T R 3 B$ in the human brain. The $p$ values for the correlation of rs3758987 and HTR3B in the frontal cortex (Fig. 3A), of rs1176744 and HTR3B in the substantia nigra (Fig. 3B), of rs4938056, and of HTR3B in the frontal cortex and hippocampus (Fig. 3C) are 0.041, $0.035,0.012$, and 0.026 , respectively.

\section{Discussion}

In the present study, through a series of association analyses including individual SNP- and haplotype-based analyses, SNP-by-SNP interaction analysis, and cismQTLs analysis on ND among 12 SNPs within HTR3A, HTR3B, and SLC6A4 in Chinese Han smokers, we found one SNP, rs1176744 in HTR3B, and one major haplotype,

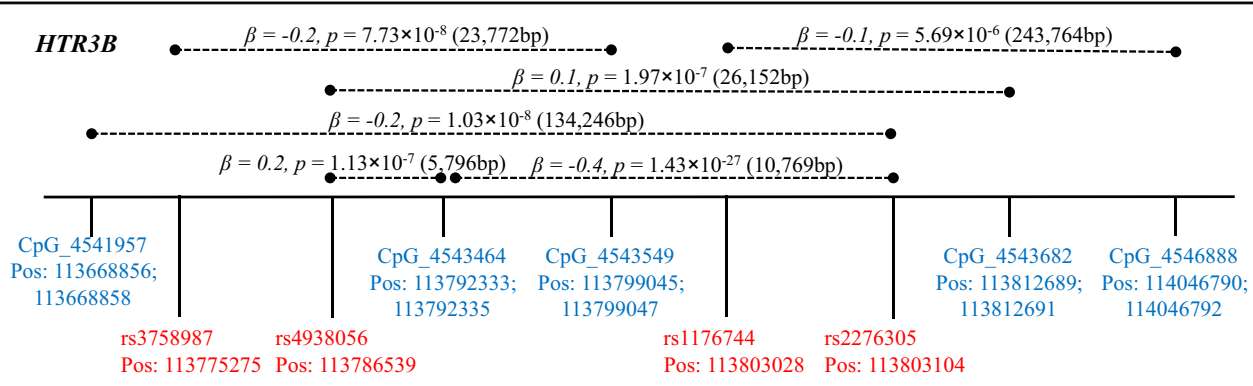

Fig. 2 Schematic diagram of cis-mQTL analysis (within $50 \mathrm{~kb}$ upstream and downstream of each SNP) and position of investigated SNPs in HTR3B. Dashed lines refer to the significant SNP-CpG pairs. Pos genome position of SNP and CpG site

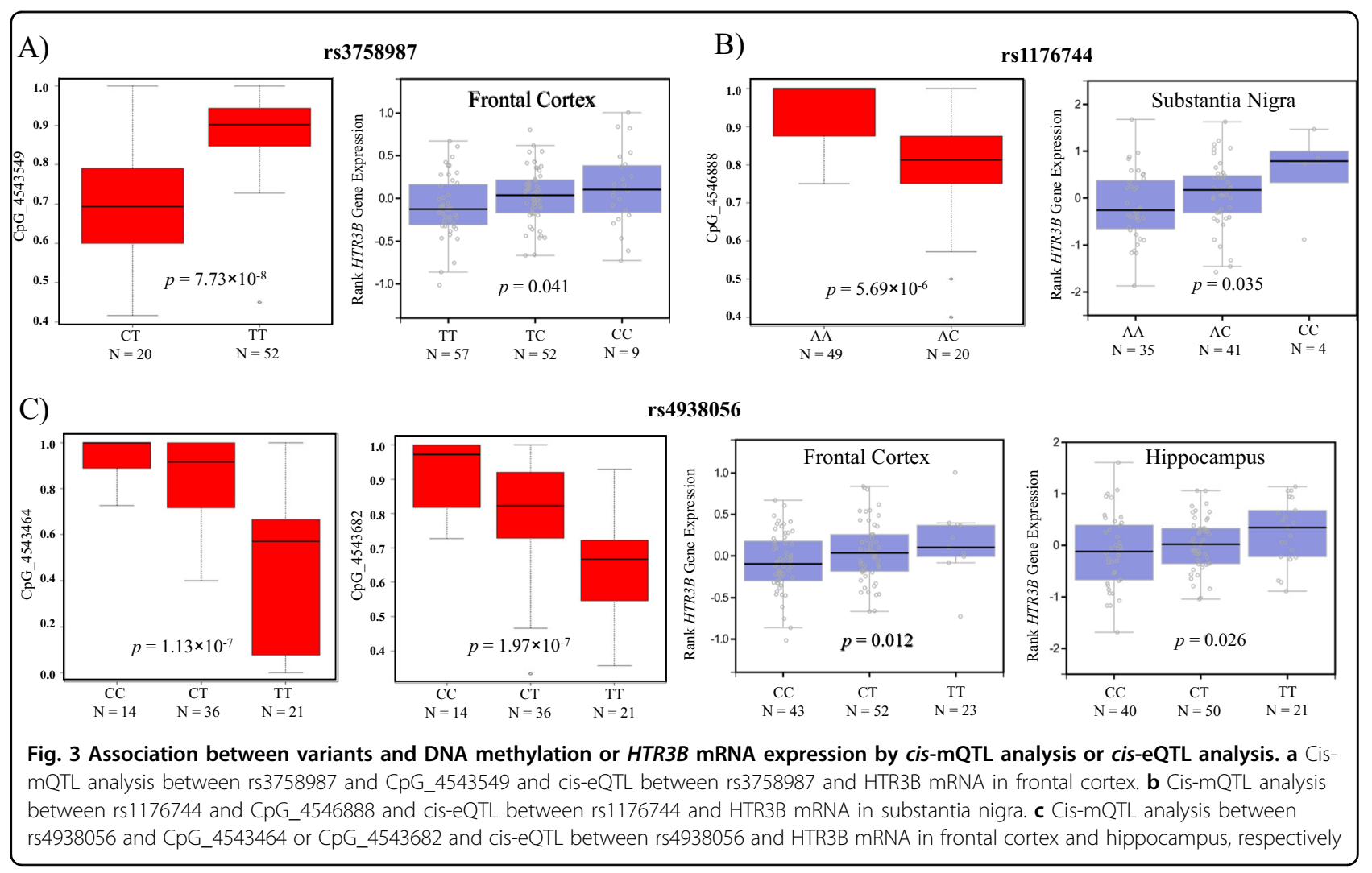


T-T-A-G, formed by rs3758987, rs4938056, rs1176744, and rs2276305 in $H T R 3 B$, to be significantly associated with the FTND score under the additive genetic model. The SNP-by-SNP interaction analysis showed that there existed significant interaction in affecting ND among SNPs rs10160548 in HTR3A, and rs3758987, rs2276305, and rs1672717 in HTR3B. We also identified four methylation sites (i.e., CpG_4543549, CpG_4543464, CpG_4543682, and CpG_4546888) associated significantly with three expression and methylation quantitative trait loci (cis-meQTL) SNPs (rs3758987, rs4938056, and rs1176744), which are located within the major haplotype detected in HTR3B.

Given that there exist various genetic architectures and smoking behaviors underlying ND in different ethnic populations ${ }^{55}$ and a high smoking prevalence in Chinese men $^{8}$, it is essential to discover the pathogenic variants of ND in the Chinese Han population. To our knowledge, this is the first study reporting the presence of significant associations of serotonergic genes with ND in Chinese Han populations. These findings highlight an important role of the variations in serotonin transporter and receptors, which govern the trans-synaptic serotonergic signaling underlying the pathophysiology of ND.

Rs1176744 is a known functional variant (Tyr129Ser) in $H T R 3 B$, which results in a change of tyrosine to serine at the 129th amino-acid residue of $5-\mathrm{HT}_{3 \mathrm{~B}}{ }^{56}$. Here we found that the rs1176744 was the only SNP showing significant association with ND at the individual level. Together with rs3758987, rs4938056, and rs2276305, they consist of a major haplotype, T-T-A-G, which is associated significantly with ND. We also found that this SNP acts as a cis-mQTL to alter the extent of methylation of CpG_4546888 in blood and cis-eQTL to modulate the mRNA expression of $H T R 3 B$ in the substantia nigra. However, no significant interactive effects of rs 1176744 with other SNPs were detected. Previously, although we showed that there exists no significant association of rs1176744 with ND in European-American (EA), AfricanAmerican (AA), and pooled samples, we did find significant interaction of this SNP with others in HTR3A and SLC6A4, indicating an important role of these genes and variants in $\mathrm{ND}^{32}$. Such inconsistent results might be attributable to the difference in allele frequencies across different populations. For instance, the MAF of rs1176744 is 0.48 for EA, 0.33 for $\mathrm{AA}$, and 0.17 for the Chinese Han population. The inconsistent MAF among different ethnic samples is rather common, which may reflect a different evolution rate and biological importance. In addition, another study of ours showed not only that SNP rs1176744 along with rs11214769 in HTR3B formed a major haplotype that is significantly associated with ND in the EA sample but also a significant interactive effect among rs1176744 in HTR3B, rs897685 and rs7118530 in
HTR3A, and rs25528 in SLC6A4 on ND in the AA sam$\mathrm{ple}^{36}$. Further, rs 1176744 proved to be significantly associated with alcohol dependence in the AA sample ${ }^{33}$. For the abovementioned results, the most reasonable explanation is the presence of heterogenity in these ethnic populations, and we for the first time show that SNP rs1176744 is active in ND in the Chinese Han population. Based on the power analysis results, we have $98.48 \%$ power to detect a polymorphism of rs1176744 (with a MAF of 0.17) with the sample size of 2616 used in this study. Although the individual SNP association analysis yielded a nominal significance of rs1176744, we had 98.48\% power to support the lack of significance.

Haplotype-based association analysis revealed that one major haplotype, T-T-A-G, constituted by rs3758987, rs4938056, rs1176744, and rs2276305 in HTR3B, was significantly associated with the FTND score $(p=$ 0.00025). Moreover, our data showed that all SNPs included in this haplotype can regulate methylation of nearby CpG sites (CpG_4543549, CpG_4543464, CpG_4543682, and CpG_4546888). The significant association of SNPs in one major haplotype with cis-mQTL indicated that the major haplotype, T-T-A-G, in HTR3B plays an important role in determining the pathology of ND through influencing the methylation of adjacent $\mathrm{CpG}$ sites in Chinese male smokers.

The three serotonergic genes, i.e., HTR $3 A$, HTR3B, and $S L C 6 A 4$, have similar functions or are known to interact $^{57}$. Thus, those with no obviously associated polymorphisms might exert genetic effects on ND when the epistatic effects are taken into consideration. In this study, by applying our own developed GMDR-GPU program ${ }^{51}$, we discovered that the best variant combination, consisting of four SNPs (rs10160548 in HTR3A, and rs3758987, rs2276305, rs1672717 in HTR3B), showed significant epistatic effects on ND $(p=0.0074)$, although the SNPs presented no important associations with ND. Previously, we showed that rs10160548 was significantly associated with SQ and heaviness of smoking index in an AA sample, and there existed significant interactive effects of this SNP with others on ND ${ }^{32}$. Furthermore, our group showed that the variants within the three genes exerted significant epistatic effects with several highly related ND assessments in EAs, AAs, and the pooled samples ${ }^{32}$. This suggests that these serotonergic genes are likely producing their effects on ND by epistasis, which might well explain why they could not be detected by association analysis.

Cigarette smoking as an exogenous modifier can change DNA methylation ${ }^{58}$, and genetic variants at specific loci can influence both regional and distant DNA methylation ${ }^{59}$. By linking genetic variants, DNA methylation, mRNA expression, and ND, we can explore the possible biological mechanisms of ND. By using a dataset with all 


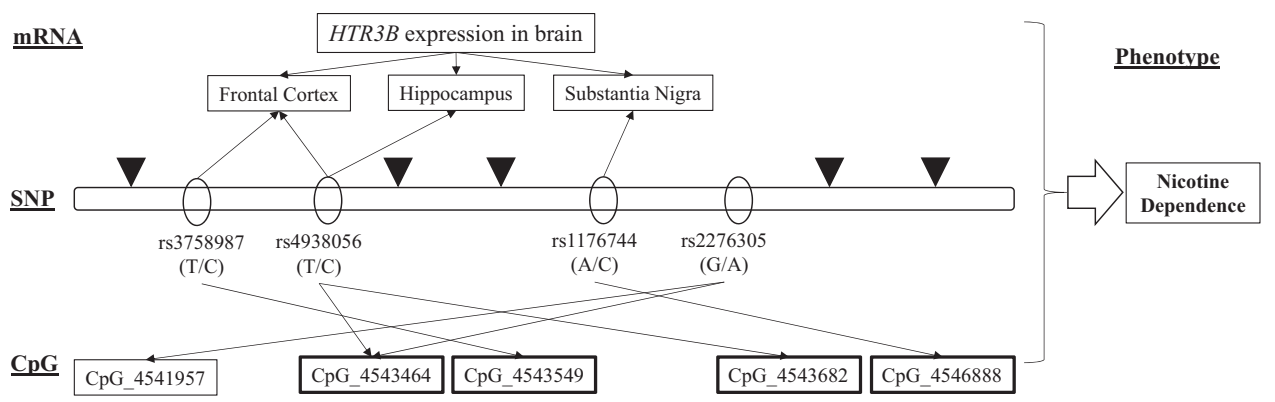

Fig. 4 Overall schematic diagram of SNPs in HTR3B, which regulates methylation loci and mRNA expression associated with smoking. Four SNPs formed as a haplotype in HTR3B significantly associated with five CpG sites. Allele-specific mRNA expression of HTR3B in the ND-related brain regions were also found, which will affect ND in Chinese Han smoker

CHRNA5 methylation, expression, and risk of SNPs data available, Hancock et al. first revealed that several genetic variations underlying CHRNA5 methylation in the human brain contributed to the risk of $\mathrm{ND}^{60}$. Recently, we linked SNP, cis-mQTL, and cis-eQTL ${ }^{45}$ and revealed two methylated sites associated significantly with three SNPs forming four significant CpG-SNP pairs in a Chinese Han population. In the present study, as shown in Fig. 4, five methylated sites associated with four SNPs in one haplotype were identified. Further, based on GTEx Portal (https://gtexportal.org/home/), a database that provides information on human gene expression and regulation and its relation to genetic variation, we identified three cis-meQTLs that affected both the extent of methylation and mRNA expression of HTR3B. Rs3758987 and rs4938056 were significantly correlated with $H T R 3 B$ mRNA expression in the human frontal cortex $(p=0.041$ and 0.012 , respectively). The frontal cortex is involved in ND, which is strongly associated with neurotransmitter release $^{61}$. We also found that rs4938056 and rs1176744 presented significant relativity with mRNA expression of $H T R 3 B$ in the hippocampus and substantia nigra, respectively. These two brain regions are known to be related to $\mathrm{ND}^{62-64}$. Thus, these SNPs may be risk targets regulating the expression of $H T R 3 B$ by altering the methylation status.

To the best of our knowledge, this is the first study to demonstrate that variants in serotonergic genes are significantly associated with ND in the Chinese Han population. From individual SNP, haplotype, and gene-gene interactions, and by linking DNA methylation and mRNA expression to perform cis-mQTL and cis-eQTL analyses, we explored the possible biological pathology involved in ND. Our results indicate that the individual SNP rs1176744 (Tyr129Ser) was nominally significant and a major haplotype formed by rs1176744, rs3758987, and rs4938056 in HTR3B showed significant association with the FTND score. Further, four methylation sites were found to be significantly associated with three cis-meQTL
SNPs. These findings extend our knowledge of the genetic roles of serotonin in affecting ND, which will help researchers look for targets that account for ND in Chinese smokers.

\section{Acknowledgements}

This study was supported in part by the China Precision Medicine Initiative (2016YFC0906300), Research Center for Air Pollution and Health of Zhejiang University, and the State Key Laboratory for Diagnosis and Treatment of Infectious Diseases of the First Affiliated Hospital of Zhejiang University. We thank Dr. David L. Bronson for excellent editing of this manuscript.

\section{Author details}

${ }^{1}$ State Key Laboratory for Diagnosis and Treatment of Infectious Diseases, The First Affiliated Hospital, Collaborative Innovation Center for Diagnosis and Treatment of Infectious Diseases, Zhejiang University School of Medicine, Hangzhou, China. ${ }^{2}$ ACT Center for Tobacco Treatment, Education and Research, Department of Otolaryngology and Communicative Sciences, University of Mississippi Medical Center, Jackson, MS, USA. ${ }^{3}$ Research Center for Air Pollution and Health, Zhejiang University, Hangzhou, China. ${ }^{4}$ Institute of Neuroimmune Pharmacology, Seton Hall University, South Orange, NJ, USA

\section{Authors' contributions}

M.D.L. conceived the study and was involved in every step of the research; $\mathrm{H}$. H., Z.Y., Y.M., W.C., and W.Y. participated clinical data collection; H.H., Z.Y., and Q. L. performed the laboratory experiments; H.H., Q.L., Y.M., L.C., and M.W.

participated in data analysis; H.H., Q.L., T.J.P., and L.J. participated in the writing of the paper. All authors approved the final manuscript.

\section{Conflict of interest}

The authors declare that they have no conflict of interest.

\section{Publisher's note}

Springer Nature remains neutral with regard to jurisdictional claims in published maps and institutional affiliations.

Supplementary Information accompanies this paper at (https://doi.org/ 10.1038/s41398-018-0290-8).

Received: 11 June 2018 Revised: 4 October 2018 Accepted: 5 October 2018 Published online: 07 November 2018

\footnotetext{
References

1. WHO. WHO Report on the Global Tobacco Epidemic, 2013: Enforcing Bans on Tobacco Advertising, Promotion and Sponsorship (World Health Organization, Geneva, 2013).
} 
2. Reitsma, M. B. F. N. et al. Smoking prevalence and attributable disease burden in 195 countries and territories, 1990-2015: a systematic analysis from the Global Burden of Disease Study 2015. Lancet 389, 1885-1906 (2017).

3. Eriksen M, Mackay J, Ross, H. The Tobacco Atlas [M]. (American Cancer Society, (2013).

4. $\mathrm{NCl} \& \mathrm{WHO}$. NCl Tobacco Control Monograph Series 21-The Economics of Tobacco and Tobacco Control (2017). http://www.who.int/tobacco/ publications/economics/nci-monograph-series-21/en/.

5. Gilmore, A. B., Fooks, G., Drope, J., Bialous, S. A. \& Jackson, R. R. Exposing and addressing tobacco industry conduct in low-income and middle-income countries. Lancet 385, 1029-1043 (2015).

6. Gowing, L. R. et al. Global statistics on addictive behaviours: 2014 status report. Addiction 110, 904-919 (2015)

7. West, R. Tobacco smoking: Health impact, prevalence, correlates and interventions [J]. Psychology \& health 32, 1018-1036 (2017).

8. Ma, Y. et al. Prevalence of cigarette smoking and nicotine dependence in men and women residing in two provinces in China. Front. Psychiatry 8, 254 (2017).

9. Do, E. \& Maes, H. Narrative review of genes, environment, and cigarettes. Ann. Med. 48, 337-351 (2016)

10. Li, M. D., Cheng, R., Ma, J. Z. \& Swan, G. E. A meta-analysis of estimated genetic and environmental effects on smoking behavior in male and female adult twins. Addiction 98, 23-31 (2003)

11. Wonnacott, S. Presynaptic nicotinic ACh receptors. Trends Neurosci. 20, 92-98 (1997).

12. Kuryatov, A., Berrettini, W. \& Lindstrom, J. Acetylcholine receptor (AChR) alpha5 subunit variant associated with risk for nicotine dependence and lung cancer reduces (alpha4beta2)(2)alpha5 AChR function. Mol. Pharmacol. 79, 119-125 (2011).

13. Wonnacott, S., Kaiser, S., Mogg, A., Soliakov, L. \& Jones, I. W. Presynaptic nicotinic receptors modulating dopamine release in the rat striatum. Eur. J. Pharmacol. 393, 51-58 (2000).

14. Partridge, J. G., Apparsundaram, S., Gerhardt, G. A., Ronesi, J. \& Lovinger, D. M. Nicotinic acetylcholine receptors interact with dopamine in induction of striatal long-term depression. J. Neurosci. 22, 2541-2549 (2002).

15. Balfour, D. J. The effects of nicotine on brain neurotransmitter systems. Pharmacol. Ther. 16, 269-282 (1982).

16. Barnes, N. M. \& Sharp, T. A review of central $5-H T$ receptors and their function. Neuropharmacology 38, 1083-1152 (1999).

17. Berger, M., Gray, J. A. \& Roth, B. L. The expanded biology of serotonin. Annu. Rev. Med. 60, 355-366 (2009).

18. Hannon, J. \& Hoyer, D. Molecular biology of 5-HT receptors. Behav. Brain Res. 195, 198-213 (2008).

19. Barnes, N. M., Hales, T. G., Lummis, S. C. \& Peters, J. A. The 5-HT3 receptor-the relationship between structure and function. Neuropharmacology 56, 273-284 (2009).

20. Connolly, C. N. \& Wafford, K. A. The Cys-loop superfamily of ligand-gated ion channels: the impact of receptor structure on function. Biochem. Soc. Trans. 32 (Pt3), 529-534 (2004).

21. Maricq, A. V., Peterson, A. S., Brake, A. J., Myers, R. M. \& Julius, D. Primary structure and functional expression of the $5 \mathrm{HT} 3$ receptor, a serotonin-gated ion channel. Science 254, 432-437 (1991).

22. McGehee, D. S. \& Role, L. W. Physiological diversity of nicotinic acetylcholine receptors expressed by vertebrate neurons. Annu. Rev. Physiol. 57, 521-546 (1995).

23. McGehee, D. S., Heath, M. J., Gelber, S., Devay, P. \& Role, L. W. Nicotine enhancement of fast excitatory synaptic transmission in CNS by presynaptic receptors. Science 269, 1692-1696 (1995).

24. Nayak, S. V., Ronde, P., Spier, A. D., Lummis, S. C. \& Nichols, R. A. Nicotinic receptors co-localize with $5-\mathrm{HT}(3)$ serotonin receptors on striatal nerve terminals. Neuropharmacology 39, 2681-2690 (2000).

25. Miyake, A., Mochizuki, S., Takemoto, Y. \& Akuzawa, S. Molecular cloning of human 5-hydroxytryptamine3 receptor: heterogeneity in distribution and function among species. Mol. Pharmacol. 48, 407-416 (1995).

26. Ramamoorthy, S. et al. Antidepressant- and cocaine-sensitive human serotonin transporter: molecular cloning, expression, and chromosomal localization. Proc. Natl Acad. Sci. USA 90, 2542-2546 (1993).

27. Hammer, C. et al. Replication of functional serotonin receptor type $3 A$ and $B$ variants in bipolar affective disorder: a European multicenter study. Transl. Psychiatry 2, e103 (2012).

28. Carver, C. S. \& Miller, C. J. Relations of serotonin function to personality: current views and a key methodological issue. Psychiatry Res. 144, 1-15 (2006).
29. Hammer, C. et al. Functional variants of the serotonin receptor type $3 A$ and $B$ gene are associated with eating disorders. Pharmacogenet. Genomics 19, 790-799 (2009).

30. Grant, K. A. The role of 5-HT3 receptors in drug dependence. Drug Alcohol Depend. 38, 155-171 (1995).

31. Watkins, S. S., Koob, G. F. \& Markou, A. Neural mechanisms underlying nicotine addiction: acute positive reinforcement and withdrawal. Nicotine Tob. Res. 2, 19-37 (2000).

32. Yang, Z. et al. Serotonin transporter and receptor genes significantly impact nicotine dependence through genetic interactions in both European American and African American smokers. Drug Alcohol Depend. 129, 217-225 (2013).

33. Enoch, M. A., Gorodetsky, E., Hodgkinson, C., Roy, A. \& Goldman, D. Functional genetic variants that increase synaptic serotonin and 5-HT3 receptor sensitivity predict alcohol and drug dependence. Mol. Psychiatry 16, 1139-1146 (2011).

34. Johnson, B. A. et al. Pharmacogenetic approach at the serotonin transporter gene as a method of reducing the severity of alcohol drinking. Am. J. Psychiatry 168, 265-275 (2011).

35. Johnson, B. A., Seneviratne, C., Wang, X. Q., Ait-Daoud, N. \& Li, M. D. Determination of genotype combinations that can predict the outcome of the treatment of alcohol dependence using the 5-HT(3) antagonist ondansetron. Am. J. Psychiatry 170, 1020-1031 (2013).

36. Yang, J. \& Li, M. D. Association and interaction analyses of 5-HT3 receptor and serotonin transporter genes with alcohol, cocaine, and nicotine dependence using the SAGE data. Hum. Genet. 133, 905-918 (2014).

37. Yang, J. \& Li, M. D. Converging findings from linkage and association analyses on susceptibility genes for smoking and other addictions. Mol. Psychiatry 21, 992-1008 (2016).

38. Li, Q., Hsia, J. \& Yang, G. Prevalence of smoking in China in 2010. N. Engl. J. Med. 364, 2469-2470 (2011).

39. Fagerstrom, K. O. Measuring degree of physical dependence to tobacco smoking with reference to individualization of treatment. Addict. Behav. 3, 235-241 (1978).

40. Heatherton, T. F., Kozlowski, L. T., Frecker, R. C. \& Fagerstrom, K. O. The Fagerstrom Test for Nicotine Dependence: a revision of the Fagerstrom Tolerance Questionnaire. Br. J. Addict. 86, 1119-1127 (1991).

41. Wei, J. et al. Association study of 45 candidate genes in nicotine dependence in Han Chinese. Addict. Behav. 37, 622-626 (2012).

42. Falush, D., Stephens, M. \& Pritchard, J. K. Inference of population structure using multilocus genotype data: linked loci and correlated allele frequencies. Genetics 164, 1567-1587 (2003).

43. Seneviratne, C. et al. Association, interaction, and replication analysis of genes encoding serotonin transporter and $5-\mathrm{HT} 3$ receptor subunits A and B in alcohol dependence. Hum. Genet. 132, 1165-1176 (2013).

44. Wen, L. et al. Significant association of the CHRNB3-CHRNA6 gene cluster with nicotine dependence in the Chinese Han population. Sci. Rep. 7, 9745 (2017).

45. Liu, Q. et al. Association and cis-mQTL analysis of variants in CHRNA3-A5, CHRNA7, CHRNB2, and CHRNB4 in relation to nicotine dependence in a Chinese Han population. Transl. Psychiatry 8, 83 (2018).

46. Krueger, F. \& Andrews, S. R. Bismark: a flexible aligner and methylation caller for Bisulfite-Seq applications. Bioinformatics 27, 1571-1572 (2011).

47. Purcell, S. et al. PLINK: a tool set for whole-genome association and population-based linkage analyses. Am. J. Hum. Genet. 81, 559-575 (2007).

48. Barrett, J. C., Fry, B., Maller, J. \& Daly, M. J. Haploview: analysis and visualization of LD and haplotype maps. Bioinformatics 21, 263-265 (2005).

49. Gabriel, S. B. et al. The structure of haplotype blocks in the human genome. Science 296, 2225-2229 (2002).

50. Schaid, D. J., Rowland, C. M., Tines, D. E., Jacobson, R. M. \& Poland, G. A. Score tests for association between traits and haplotypes when linkage phase is ambiguous. Am. J. Hum. Genet. 70, 425-434 (2002).

51. Zhu, Z. et al. Development of GMDR-GPU for gene-gene interaction analysis and its application to WTCCC GWAS data for type 2 diabetes. PLOS ONE 8 , e61943 (2013).

52. Traylor, M. et al. Genetic variation at $16 \mathrm{q} 24.2$ is associated with small vessel stroke. Ann. Neurol. 81, 383-394 (2017).

53. McClay, J. L. et al. High density methylation QTL analysis in human blood via next-generation sequencing of the methylated genomic DNA fraction. Genome Biol. 16, 291 (2015).

54. Shabalin, A. A. Matrix eQTL: ultra fast eQTL analysis via large matrix operations. Bioinformatics 28, 1353-1358 (2012). 
55. Herzog, T. A. \& Pokhrel, P. Ethnic differences in smoking rate, nicotine dependence, and cessation-related variables among adult smokers in Hawaii. J. Community Health 37, 1226-1233 (2012).

56. Walstab, J., Hammer, C., Bonisch, H., Rappold, G. \& Niesler, B. Naturally occurring variants in the HTR3B gene significantly alter properties of human heteromeric 5-hydroxytryptamine-3A/B receptors. Pharmacogenet. Genomics $\mathbf{1 8}$ 793-802 (2008).

57. Herman, A. I. \& Balogh, K. N. Polymorphisms of the serotonin transporter and receptor genes: susceptibility to substance abuse. Subst. Abuse Rehabil. 3, 49-57 (2012).

58. Breitling, L. P., Yang, R., Korn, B., Burwinkel, B. \& Brenner, H. Tobacco-smokingrelated differential DNA methylation: $27 \mathrm{~K}$ discovery and replication. Am. J. Hum. Genet. 88, 450-457 (2011).

59. Lee, K. W. \& Pausova, Z. Cigarette smoking and DNA methylation. Front. Genet. 4, 132 (2013)
60. Hancock, D. B. et al. A multiancestry study identifies novel genetic associations with CHRNA5 methylation in human brain and risk of nicotine dependence Hum. Mol. Genet. 24, 5940-5954 (2015).

61. Markou, A. Review. Neurobiology of nicotine dependence. Philos. Trans. R. Soc Lond. B Biol. Sci. 363, 3159-3168 (2008).

62. Davis, J. A. \& Gould, T. J. Associative learning, the hippocampus, and nicotine addiction. Curr. Drug Abuse Rev. 1, 9-19 (2008).

63. Subramaniyan, M. \& Dani, J. A. Dopaminergic and cholinergic learning mechanisms in nicotine addiction. Ann. N. Y. Acad. Sci. 1349, 46-63 (2015).

64. Keath, J. R., lacoviello, M. P., Barrett, L. E., Mansvelder, H. D. \& McGehee, D. S. Differential modulation by nicotine of substantia nigra versus ventral tegmental area dopamine neurons. J. Neurophysiol. 98, 3388-3396 (2007). 